\title{
The Use of Group Therapy as a Means of Facilitating Cognitive-Behavioural Instruction for Adolescents With Disruptive Behaviour
}

\author{
Stephen Larmar \\ Griffith University, Australia
}

$\mathrm{T}^{\text {mimaina }}$

eports on the findings of an action research enquiry examining the efficacy of group therapy as a means of facilitating cognitive-behavioural instruction for students who exhibit disruptive behaviours. A curriculum comprising the key tenets of cognitive-behaviour modification was developed and taught over a 9-week period to a group of 12 Year 7 adolescent students selected from a primary school in the Brisbane metropolitan district, Queensland, Australia. Six of the participants for the investigation were identified as 'at-risk' of engaging in disruptive behaviours based on extensive observations from members of the school administration team. The remaining students served as role models of prosocial behaviours throughout the intervention. Each session incorporated group discussions and physical challenges that were formulated to facilitate investigation of key concepts of cognitive-behavioural therapy. The findings that emerged from the investigation provide support for the use of group therapy for this form of intervention.

Child and adolescent disruptive behaviour is a phenomenon that has had an increasingly negative impact on schools and the wider community (August, Realmuto, Hektner, \& Bloomquist, 2001; Frick, 2004). To address the rise in student disruptive behaviour in educational settings, a range of interventions have been developed that are tailored to the individual needs of the student (August, Egan, Realmuto, \& Hektner, 2003; Conduct Problems Prevention Research Group, 2002; Little \& Hudson, 1998).

Researchers have examined the efficacy of various approaches designed to elicit change in disruptive behaviour exhibited by school students (August et al., 2001;

Address for Correspondence: Dr Stephen Larmar, School of Human Services, Griffith University, Logan Campus, University Drive, Meadowbrook QLD 4131, Australia. E-mail: s.larmar@griffith.edu.au 
Hoff \& DuPaul, 1998; Little \& Hudson, 1998; Walker, Kavanagh, Stiller, \& Golly, 1998). A substantial body of literature indicates that cognitive-behavioural techniques have been effective in addressing problem behaviours (Albano et al., 2004; Erickson, 1998; Feindler \& Scalley, 1998; Kendall \& Panichelli-Mindel, 1995). Researchers have also identified that group therapy is an effective modality for facilitating cognitive-behavioural instruction for children and adolescents exhibiting behaviour problems (Feindler \& Scalley, 1998; Henin, Warman, \& Kendall, 2002; Kastner, 1998).

Prior research has given considerable attention to the study of outcomes of cognitive-behavioural instruction within the context of group therapy (Albano, Krain, Podniesinski, \& Ditkowsky, 2004; Free, 1999; Kastner, 1998; Kendall \& PanichelliMindel, 1995). However, the study of the dynamics emerging during facilitated group therapy has been largely neglected. This investigation centres on the processes involved in the use of group therapy to facilitate cognitive-behavioural instruction for adolescent students who exhibit disruptive behaviours.

\section{Child and Adolescent Disruptive Behaviour: Implications for Schools}

The rise in disruptive responses in children and adolescents is of serious concern to personnel working in schools (Little \& Hudson, 1998). Researchers investigating the phenomenon of problem behaviour have recognised the negative implications for students who frequently exhibit intense, disruptive responses (Frick, 2004; Little \& Hudson, 1998). With reducing parental and familial involvement in the child and adolescent development process, educationalists are recognising the proactive role a school must take in assisting students who struggle to manage their behaviours effectively (Little \& Hudson, 1998).

The literature strongly suggests that the occurrence of disruptive behaviour in children and adolescents is highly correlated to deficiencies in cognition and social problem-solving abilities (Erickson, 1998; Kendall \& Panichelli-Mindel, 1995; Taylor \& Novaco, 2005). Common responses observed in children and adolescents with disruptive behaviour include verbal and physical conflict with peers, ignoring the rights of others, throwing tantrums, and behaviours indicating poor social skills and lack of self-control (Kendall \& Panichelli-Mindel, 1995; Renfrew, 1997).

It is considered that school-based interventions for students with problem behaviour are advantageous, as students are already engaging in experiences in the school context that assist them in their social and cognitive development (Feindler \& Scalley, 1998). Stoiber and Kratochwill (1998) acknowledge the effectiveness of schools in addressing maladaptive behaviours given that prevention and treatment programs are often readily accessible to the student. Greenberg, Domitrovich and Bumbarger (2001) emphasise that systemic and effective ways for schools to intervene in the behaviour of those youth most at risk for continuing or later antisocial behaviour are necessary. As disruptive behaviours in children and adolescents become more apparent school personnel must work to develop intervention techniques that foster more appropriate responses in students with difficulties.

Given the significant correlation between problem behaviours in children and adolescents and deficiencies in cognition and social problem-solving abilities, cognitive-behavioural approaches are deemed highly effective in assisting children and adolescents with behavioural difficulties (Erickson, 1998; Kendall \& 
Panichelli-Mindel, 1995; Taylor \& Novaco, 2005). Further, the use of group therapy as a means to facilitate cognitive-behavioural instruction is also advantageous given children and adolescents' propensity towards socialisation. The following section provides an overview of cognitive-behavioural therapy and group therapy as cogent approaches for working with children and adolescents with problem behaviours.

\section{Cognitive-Behavioural Therapy and Group Therapy: An Overview}

Cognitive-behavioural therapy is defined as a form of intervention that is concerned with reducing maladaptive behaviours through the identification and modification of apparent distorted patterns of thinking (Free, 1999). Practitioners of cognitivebehavioural therapy work collaboratively with their clients focusing on the areas of behaviour, cognition and affect to facilitate changes in behaviour (Schuyler, 2003).

The process concerns itself with both individuals' external environments and their internal processing of the world. Interventions involving cognitive-behavioural therapy build on existing known principles of behavioural therapy. The cognitive-behavioural therapist examines external behaviours, recognising the significance of client cognition in the process of behaviour change (Schuyler, 2003). Through the process of therapy the therapist encourages the client to analyse maladaptive thought processes to assist the client in identifying faulty patterns of thinking. The client is further encouraged to replace such thought patterns with rationally centred cognitions (White, 2000).

Group therapy is considered to be a successful intervention treatment for child and adolescent populations (Hoag \& Burlingame, 1997; Johnson et al., 1998; White, 2000). The process of group therapy is known to elicit changes in the areas of client affective, cognitive and behavioural functioning (Stoiber \& Kratochwill, 1998). The inherent propensity of children and adolescents towards socialising makes group therapy a salient model of intervention for working with this population. Researchers of group therapeutic processes acknowledge that children and adolescents can benefit developmentally from relationships formed with peer groups (Mishna \& Muskat, 1998). While not discounting individual therapeutic approaches, it is suggested within the context of group therapy children and adolescents can engage in an experience of belonging that can facilitate them examining the nature of their interpersonal problems while realising the possibilities and individual meanings of interpersonal change (Kastner, 1998).

\section{Method}

A group-based intervention was facilitated by the researcher/practitioner as a means of investigating the dynamics that emerged through cognitive-behavioural instruction for adolescent students with disruptive tendencies. It should be noted that the researcher/practitioner is a trained counsellor who has had extensive experience working with children and families at-risk. Further, the researcher/practitioner has had a breadth of experience providing training for school guidance counsellors and psychologists in primary, secondary and tertiary settings. In this capacity, the researcher/practitioner was able to competently undertake the role of facilitating cognitive-behavioural instruction within a group therapy context. 
The group comprised seven male and five female Year 7 students drawn from a selected school in the Brisbane Metropolitan area. Barrett, Lowry-Webster and Holmes (1999) recommend the use of small groups comprising 8 to 12 participants for this form of intervention. They assert that individuals learn most effectively by observing and assisting others. Within this context, participants are also provided opportunities to practise newly acquired skills in a safe and supportive environment.

The deputy principal of the school screened each student in collaboration with the researcher/practitioner to determine subject suitability for the investigation. The screening process involved the identification of children from the current Year 7 cohort whose behaviours matched a descriptor of a child who typically exhibits externalising behaviour problems. Six students (four male and two female students) were identified who closely matched the descriptor based on observed disruptive behaviours in the classroom and playground that placed them at risk of school failure. The remaining six subjects were identified as students who were popular among their peers and whose behaviours were considered exemplary. These students acted as role models throughout the intervention. Participants targeted for the study were given the choice of involvement in the investigation. Parental and departmental consent was also sought prior to the commencement of the intervention. The targeted students were informed of the researcher's/practitioner's intention to collect and retain the students' written reflections at the conclusion of each session.

A curriculum comprising the key tenets of cognitive-behavioural therapy was developed by the researcher/practitioner and was based on an adaptation of Kaplan and Carter's (1995) process of cognitive-behaviour modification. The curriculum facilitated participant focus on covert behaviours such as thoughts and feelings. The researcher/practitioner devised a booklet containing written activities that explores key concepts of Kaplan and Carter's cognitive-behaviour modification program. Learning experiences were developed to explore key principles such as the impact of beliefs on an individual's behaviour, recognising thoughts associated with an event that impact on an individual's responses and the development of strategies to assist individuals in choosing pro-social behaviours in times of conflict. The curriculum was designed to cater to the age span of the target group and organised sequentially to allow for the instruction to be delivered on a weekly basis over nine weeks. Teaching techniques that encouraged group interaction were incorporated into the proposed curriculum design. These included a series of physical games and challenges that explored concepts that were linked to principles of cognitive-behavioural instruction.

The group met at a designated time each week for a period of 9 weeks. The duration of each meeting was approximately one and a half hours. Barrett et al. (1999) suggest that short-term intervention models are best facilitated over an 8- to 12week period with participants meeting at least once a week for optimal effectiveness. Group meetings were held in an appropriate space at the school selected at the discretion of the deputy principal. Students who participated in the group were freed from regular school activities during the period allocated for the intervention meetings.

Action research was selected as the research paradigm for the investigation. Action research draws on qualitative methods and the multiple perspectives of educational ethnography (Zeni, 1998). The primary goal of the action researcher is to 
investigate how to improve existing practice (Stringer \& Dwyer, 2005). This study involved the researcher/practitioner actively engaging in the research setting to inform existing practices. Action research facilitated this process and enabled the researcher/practitioner to become an active participant within the research setting. Within the framework of action research the researcher/practitioner engaged in the investigation as an active participant to experience and derive meaning from the dynamics associated with the processes of group therapy and cognitive-behavioural instruction.

A series of research questions were identified to guide the course of the investigation. These questions included:

- What are the researcher's/practitioner's responses to the processes implemented in the use of group therapy?

- What is the researcher's/practitioner's reflection of the responses identified in the process of implementing cognitive-behavioural instruction?

- What are the researcher's/practitioner's reflection and responses to the relational exchanges that take place between the group participants?

Methods of data collection included research participant field notes of the nine sessions represented within the framework of an action research cycle. They were considered in conjunction with the related research questions formulated for the study. In addition, student responses to a series of questions were recorded that were germane to the action research component of the investigation. The particular items of questions included:

- What did you think of the session/how did you feel it went?

- What aspects of the session went well?/What would you keep the same?

- What would you change about the session?/What would you do differently?

- What would you like to focus on in the next session?/What would you like to do more of?

The researcher/practitioner subjected himself to the same body of questions that were given to the participants in the study. Following the gathering of this information, the data were discussed on a weekly basis with an external experienced action researcher engaged in higher education to establish patterns of deviation and congruence. In addition, this evaluation through a third party assisted in necessary modifications to the organisational enhancement of the outcomes of the following weeks' sessions. Figure 1 provides a diagrammatic representation of the researcher's/practitioner's engagement in the group therapy intervention including data collection and ongoing analysis of the facilitated intervention.

Data were analysed within a framework of interpretational analysis to identify regularities in patterns of both individual behaviour and interactions between participants. The focus of the analysis centred on the effectiveness of processes utilised during the course of the intervention. Information was gathered from four primary sources. The first source was the research participant's field notes. The second 

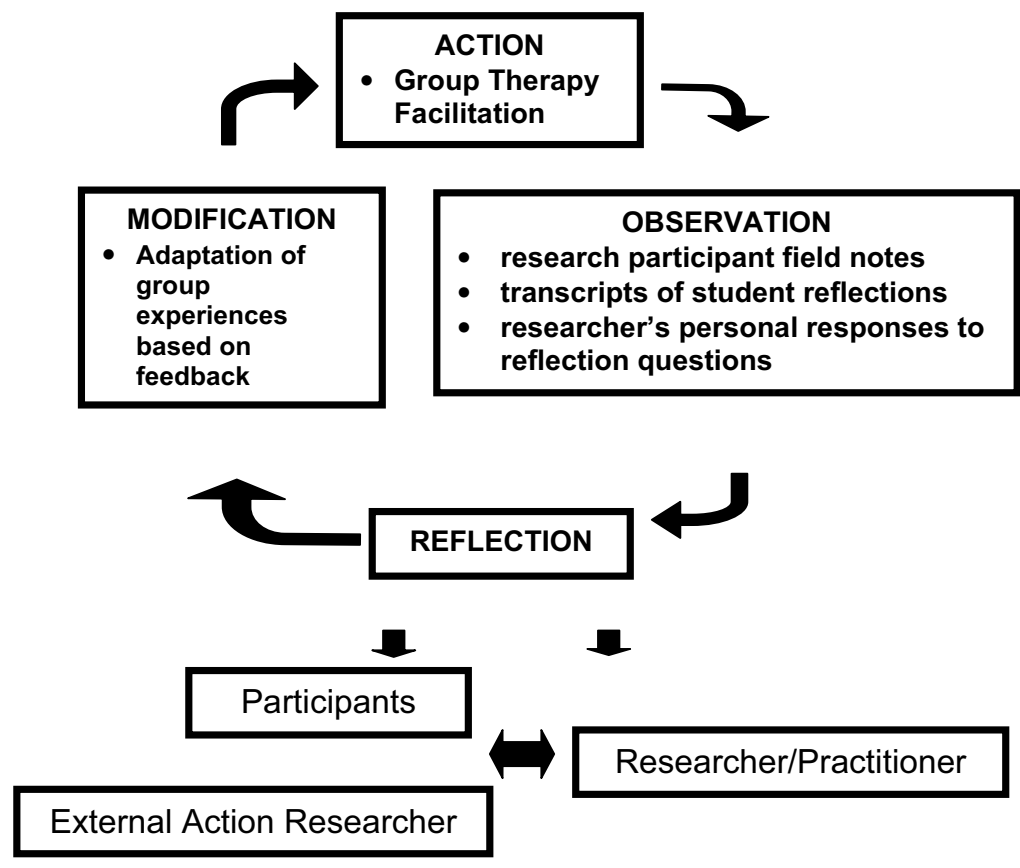

FIGURE 1

Action research reflection cycle.

consisted of nine action research cycles relating to each session formulated by the researcher/practitioner. These documented the series of actions, observations, reflections and modifications pertinent to each session. The third source of information was the transcriptions of responses to questions formulated and addressed by the researcher/practitioner at the conclusion of each session. The final source was the collected written responses of the student participants to the same body of questions.

The body of information was reorganised to present a summary of key findings that emerged throughout the investigation. A final action research summary was formulated to diagrammatically represent this information. In conjunction with this summary, a list of key findings that evolved during the process of addressing the related research questions was also developed. From this several constructs and propositions relating to the success of the facilitation of cognitive-behavioural therapy within the group context emerged.

Data were analysed to inform the action research process by highlighting the need for modifications and reinforcement in the research spiral of activities. In addition, it identified regularities in patterns of both individual behaviour and interactions between participants. The development of the series of action research cycles, including the final summary provided a synthesis of the intervention process, indicated frequently emerging topics that related to social exchanges and participant 
responses observed during the course of the intervention. The formulation of the final action research summary and the concluding responses directed from the related research questions was a culminating activity of the study.

At the conclusion of the intervention, through this system of organisation the researcher/practitioner recontextualised the information identifying connections between patterns of behaviour and the functioning of the group. This facilitated an assessment of the effectiveness of procedures. In this way the researcher/practitioner developed conclusions relating to the organisation of this form of intervention that will inform future practice. Additionally, issues relating to group processes could also be examined to determine procedures necessary for the successful facilitation and implementation of group interventions focussing on cognitive-behavioural techniques.

\section{Results}

The following section provides a summary of responses that indicate the effectiveness of the intervention and the choice of group therapy as an appropriate modality for the facilitation of cognitive-behavioural instruction for adolescents at-risk.

\section{Research Question One Summary}

The first related research question focused on the appropriateness of processes selected for the various sessions: What are the researcher's/practitioner's responses to the processes implemented in the use of group therapy?

Through the researcher's/practitioner's consideration of the first research question a series of key processes were identified that provided a framework for each session. These were physical activities, group discussion and strategies of management. A summary of the findings significant to these processes follows.

\section{Physical Activities}

The incorporation of physical activities in the framework of each session was significant to the successful facilitation of the intervention. The effectiveness of these activities was made evident through the reflections of the participant responses. Activities included physical games and challenges that engaged all participants. The following has been identified as being significant:

- The balance of physical activities and discussion proved effective throughout the entire course of the intervention — participants enthusiastically engaged in the various activities and individual interest was maintained throughout each session.

- The incorporation of activities that encouraged full group participation was effective in promoting inclusivity and acceptance - the researcher's/ practitioner's observations indicated that participants generally made a concerted effort to cooperate with one another throughout each session.

- The inclusion of physical activities to explain theoretical concepts relating to cognitive-behavioural instruction seemed highly effective in linking participant understanding to such concepts - due to the general enthusiasm 
that was elicited through engagement in the physical activities, participants seemed to link theoretical concepts more readily because of the positive experience associated with the activity.

\section{Group Discussion}

Throughout the intervention a series of group discussions were facilitated that focused on key principles of cognitive-behavioural instruction. Each discussion was conducted either on the floor or around a large table with participants forming a circle to encourage individual focus and group inclusivity. The organisation of each discussion was considered with a view to eliciting meaningful engagement and interaction for all participants. A summary of findings is presented below:

- The use of age-appropriate, personal examples to illustrate concepts of cognitive-behavioural instruction was effective in assisting participants to comprehend concepts explored.

- The use of the booklet in conjunction with the group discussion proved effective in assisting participants in their understanding of key concepts - the combination of learning experiences accommodated a range of individual learning styles.

- The use of a whiteboard during the discussion of writing tasks was effective in helping the group focus on the activity and facilitated the sharing of individual responses - this strategy, further, provided visual cues that assisted in encouraging participant focus.

\section{Strategies of Management}

The researcher/practitioner considered a series of management strategies that were implemented in the intervention. These strategies served to promote both individual and group cooperation and were successful overall in eliciting participant prosocial behaviours and the maintenance of group order and homogeneity. The management strategies were either preventative or reactive in the event that undesirable behaviours surfaced during the course of the intervention. The following has been identi-

- The researcher's/practitioner's democratic approach to the facilitation of each session was effective in eliciting individual and group cooperation - the researcher's/practitioner's observation of participant's conciliatory responses to his instruction indicated that student participants felt comfortable with the adopted style of management of the group.

- The formulation and incorporation of group goals (such as listening to the contributions of others, respecting the rights of others, communicating positively to all participants of the group) to provide a framework for communicating behavioural expectations for each session was effective in encouraging prosocial behaviours - it provided students with continual reminders that assisted in reinforcing positive behaviours deemed necessary for the facilitation of group cooperation. 
- The initial focus on group goals and behavioural expectations at the commencement of each session was effective in focusing the group and reminding participants of the group's expectations regarding behaviour and participation.

- Seating participants at risk of group exclusion (due to inappropriate behaviour) in close proximity to the researcher/practitioner assisted them in maintaining focus and choosing prosocial behaviours.

- The development of a contingency plan (strike system) for extreme inappropriate behaviours was effective in preserving the sense of order and encouraging full participant cooperation.

- Running each session prior to the participants' main lunch break was preferable to running the session following lunch - participants appeared generally more focused during morning sessions in contrast to afternoon sessions where certain students were more disruptive.

- The incorporation of activities that required participants to work in smaller groups was effective in facilitating peer support for individuals who were struggling to complete activities on their own - the participants identified as 'supporters' appeared enthusiastic in this role.

- The incorporation of 'noninvasive' management strategies that provided cues for the redirection of minor disruptions were effective in maintaining group order and eliciting positive behavioural choices - these strategies also served to minimise the potential for negative attention to be focused on the disruptive individual.

- Communicating expectations prior to the commencement of a new activity facilitated participant focus and comprehension of behavioural and task guidelines - this strategy served to minimise disruptive behaviours during each activity.

\section{Research Question Two Summary}

The second related research question focused on the participants' responses to the processes implemented throughout the intervention. What is the researcher's/practitioner's reflection of the responses identified in the process of implementing cognitive-behavioural instruction?

Overall the participants responded favourably to the intervention with the majority indicating that they benefited from involvement in the sessions. Key findings relating to participant responses to processes employed are summarised below:

- The majority of participants consistently indicated that they enjoyed each session.

- The majority of participants communicated their enjoyment of the selection of physical activities organised as part of the overall intervention framework - participant enthusiasm for the various physical activities was evident in the researcher's/practitioner's observations of participants. 
- At the conclusion of each session the majority of participants indicated that they wished to change nothing about the content of each session - the recommendations for change were, generally, unrealistic such as making the session longer, repeating preferred activities excessively or permanently removing an individual from the group.

- Most participants indicated that the balance of physical activities and discussion seemed appropriate.

- All participants communicated that they had learnt some useful strategies that they believed could be personally implemented.

- All participants were particularly enthusiastic about working as a group - no participant indicated a preference for working on their own.

- Some participants who communicated positive sentiments about working in the group suggested that the behaviour of some students negatively affected the positive group dynamic that had developed during the intervention.

\section{Research Question Three Summary}

The third question centred on the researcher's/practitioner's observations of relational exchanges that took place between participants during the course of the intervention. What are the researcher's/practitioner's reflection and responses to the relational exchanges that take place between the group participants?

Through examination of the third related research question two key categories emerged. The first focused on the relational exchanges that occurred between the student participants. The second examined the dynamics that resulted from the relational exchanges between the student participants and the researcher/practitioner. A summary of findings relating to the various relational responses follows.

\section{Student Participant Relational Exchanges}

Student participants interacted positively overall, the majority exhibiting behaviours that promoted acceptance and facilitated an inclusive dynamic. Two participants occasionally displayed behaviours that affected positive dynamics of the group. One participant, who struggled to find acceptance from the wider group, only attended the first three sessions. A summary of the researcher's/practitioner's key observations relating to student participant interaction follows:

- Student participants generally worked cooperatively throughout the course of the intervention - this was evidenced in the researcher's/practitioner's observations of the group during each session.

- Two participants were particularly antagonistic towards the wider group, however, both responded promptly to behaviour management strategies implemented.

- The group dynamic significantly changed during sessions where one of the antagonistic participants was absent - the group was more settled and the level of cooperation among group members improved considerably. Discussion with the group focusing on reasons why the group behaved 
differently during times of particular participant's absence may have been of benefit - the group may have considered strategies that served to reduce the negative affects of participant misbehaviour on the group dynamic.

- Certain participants attempted to dominate some of the group discussions during particular sessions, however, strategies that ensured discussion was equitably shared among all participants proved effective.

- Participants became more accepting of group members as the intervention progressed (with the exception of two individuals) which created a greater sense of group cohesion and unity - discussion with the wider group focusing on the inclusion of the less accepted participants may have encouraged greater acceptance of these individuals.

- The exchange between male and female participants improved markedly as the intervention progressed - the various activities selected for the intervention that focused on peer cooperation seemed to influence the positive change in male and female relational exchanges.

- Overall, participants worked effectively in groups of mixed gender and in combination with individuals outside of immediate peer groupings, with the exception of the two participants who affected the positive dynamic of the wider group - the activities selected were effective in facilitating group homogeneity, however, further discussion with participants may have influenced changes in the behaviour of the accepted group members to more readily embrace those individuals who were being ostracised from the wider group.

- Overall, the male participants tended to try to dominate activities and discussion throughout the intervention - the researcher/practitioner continued to utilise management strategies that served to alleviate the problem of male participants dominating the sessions.

- Participants' positive responses to other group members' input seemed to facilitate greater group cohesion - the researcher/practitioner continually encouraged participants to encourage the sharing of other individuals' ideas.

- Two male participants were antagonistic towards one another in earlier sessions - by keeping these individuals separated in future sessions antagonistic behaviours ceased.

- Certain participants regularly expressed their frustration at the antagonistic individuals' attempts at destroying the positive dynamics that had been established between the remainder of the group members - individual discussion may have proved constructive with these participants, allowing them to express their frustrations openly and to consider strategies that could decrease the negative affects such individuals were having on the group.

- During selected physical activities some minor arguments arose regarding students not adhering to the rules (e.g., cheating etc.), however, the stu- 
dents appeared to work cooperatively overall and seemed to enjoy working as a team - the researcher/practitioner often assumed the role of mediator to deal with contentious issues as they arose and found this approach to be effective in defusing some of the frustrations associated with participant disputes.

\section{Student-Researcher/Practitioner Relational Exchanges}

A positive working relationship developed between the student participants and the researcher/practitioner by the conclusion of the first session. The researcher/practitioner chose a democratic approach to the facilitation of the intervention. This served to establish and maintain a positive dynamic between students and the researcher/practitioner throughout the study. The researcher/practitioner observed that particular strategies of management and communication were effective in eliciting student confidence in the researchers/practitioner's leadership and encouraging prosocial behaviours among the group. The following key findings are presented as a summary, identifying the researcher's/practitioner's influence on the group:

- The use of a 'strike' system that served to provide cues to redirect misbehaviour worked as an effective tool of management and assisted in maintaining positive relationships between group participants and the researcher/practitioner.

- All participants related positively with the researcher/practitioner overall throughout the course of the intervention which assisted in the researcher/ practitioner maintaining a democratic approach to the facilitation of the group.

- The researcher's/practitioner's positive communication throughout the intervention served to establish trust and communicate acceptance to all members of the group.

- The researcher's/practitioner's use of encouragement following meaningful participant responses during group discussions served to reinforce the behaviour, encouraging participants' contributions to future discussions.

- The researcher/practitioner encouraging more reserved participants to share their ideas established a dynamic that facilitated equitable communication of individual's thoughts and opinions throughout the course of the intervention.

- The researcher's/practitioner's encouragement of positive behavioural choices directed at participants whose behaviour had placed them at risk of exclusion in earlier sessions positively affected their behaviours in remaining sessions.

- The researcher's/practitioner's communication of behavioural guidelines at the beginning of each session and the reiteration of group goals provided a structured framework and equitable forum for participants to relate in. 
- The researcher's/practitioner's inclusion of clear and concrete examples to illustrate points of discussion assisted in increasing student comprehension throughout the intervention.

- The researcher's/practitioner's assertive and direct confrontation when dealing with participants speaking out of turn or speaking disrespectfully elicited prompt changes in behaviour.

- Continual reinforcement of group goals and behavioural guidelines encouraged focus and cooperation during engagement in the various activities.

\section{Discussion}

The findings that have emerged throughout the course of the investigation indicate that group therapy is an effective means of facilitating cognitive-behavioural instruction for students exhibiting disruptive behaviours. Through a textual analysis of the students' comments it was perceived that the student participants found the experience of working as a group to be instrumental in facilitating a positive learning experience where key concepts of cognitive-behavioural therapy could be explored. The group context also provided a framework in which a broader range of learning experiences could be incorporated to further enhance this form of intervention.

In addressing the related research questions throughout the course of the investigation, key methodologies and processes that assisted in the successful implementation and facilitation of the group therapy process became evident. The group gender mix, the timing and organisation of the various sessions, the researcher's/practitioner's democratic approach to the facilitation of each session, the use of the work booklet and the incorporation of the physical activities and discussion were brought together in a nonthreatening, naturalistic setting to bring about positive, cohesive responses.

it is impossible to deconstruct all the elements of the intervention process. a key proposition that emerged during the investigation indicated that the processes and organisational structure of the intervention synergistically created a positive outcome that reinforced the effectiveness of the group therapy process.

\section{Limitations}

A number of limitations have been identified in respect to the methodologies applied in the framework of the research design. The first limitation of the investigation focused on the researcher's/practitioner's current practice in cognitive-behavioural instruction. This confines its application to the work of others serving in a similar field. Second, the study's focus on a specific age group makes its findings unable to be generalised to students in other age groups. Third, the specificity of the demographic location of the school limits the generalisability of the findings to schools in other noncomparative demographic locations. Fourth, the researcher/practitioner was not acting as an independent observer, but as a facilitator-teacher and researcher/practitioner. This dual role may be seen to act as an inhibitor for observed outcomes. Finally, the duration of the study was limited to nine one-hour 
weekly sessions. This framework limited observations to a restricted time frame, therefore inhibiting its generalisability to longitudinal investigations.

\section{Future Research}

This study has proven to be effective in demonstrating the effectiveness of group therapy in facilitating cognitive-behavioural instruction for adolescents at risk. However the study has been limited to a single participant observer with a defined age group, gender balance and socioeconomic mix. Future research needs to confirm the efficacy of this process by:

- undertaking the process over a greater time frame

- engaging different schools and demographic locations

- studying the dynamics of this process with different age groups

- exploring the effects of group therapy for participants from same gender groups

- incorporating independent observers into the action research methodology — identifying key observations independent of the personal biases of the participant researcher/practitioner

- facilitating group therapy processes with alternative culture groups.

In addition, future studies in this area can be further advanced through the implementation of longitudinal studies in contrast to the current cross-sectional design.

\section{Conclusion}

This article has presented findings from a recent study that supports the efficacy of group therapy as a means of facilitating cognitive-behavioural instruction for addressing maladaptive behaviours in children. Key issues associated with management of disruptive behaviours in children in regular school settings were identified. A description of the design and methodologies adopted for the purposes of facilitating the study under examination was outlined. Key findings that emerged through the facilitation of the investigation were identified. Finally, limitations associated with the study's design were acknowledged and considerations for future research in the area were sign posted to direct further research agendas in group therapy and cognitive behavioural instruction.

\section{References}

Albano, A.M., Krain, A.L., Podniesinski, E., \& Ditkowsky, K.S. (2004). Cognitive-behavior therapy with children and adolescents. In J.H. Wright (Ed.), Cognitive-behavior therapy, review of psychiatry (Vol. 23). Washington, DC: American Psychiatric Publication.

August, G.J., Egan, E.A., Realmuto, G.M., \& Hektner, J.M. (2003). Parcelling component effects of a multifaceted prevention program for disruptive elementary school children. Journal of Abnormal Child Psychology, 31(5), 515-527. 
August, G.J., Realmuto, G.M., Hektner, J.M., \& Bloomquist, M.L. (2001). An integrated components preventative intervention for aggressive elementary school children: The early risers program. Journal of Consulting and Clinical Psychology, 69(4), 614-626.

Barrett, P., Lowry-Webster, H., \& Holmes, J. (1999a). FRIENDS group leader's manual for children. Brisbane, Australia: Australian Academic Press.

Barrett, P., Lowry-Webster, H., \& Holmes, J. (1999b). FRIENDS workbook for children. Brisbane, Australia: Australian Academic Press.

Conduct Problems Prevention Research Group. (2002). Evaluation of the first 3 years of the fast track prevention trial with children at high risk for adolescent conduct problems. Journal of Abnormal Child Psychology, 30(1), 19-35.

DiGiuseppe, R., Tafrate, R., \& Eckhardt, C. (1994). Critical issues in the treatment of anger. Cognitive and Behavioral Practice, 1, 111-132.

Erickson, M.T. (1998). Behavior disorders of children and adolescents: Assessment, etiology, and intervention (3rd ed.). Englewood Cliffs, NJ: Prentice-Hall.

Feindler, E.L., \& Scalley, M. (1998). Adolescent anger-management groups for violence reduction. Handbook of group intervention for children and families. Boston: Allyn and Bacon.

Free, M.L. (1999). Cognitive therapy in groups: Guidelines and resources for practice. New York: John Wiley.

Frick, P.J. (2004). Developmental pathways to conduct disorder: Implications for serving youth who show severe aggressive and antisocial behaviour. Psychology in the Schools, $41(8), 823-834$.

Greenberg, M.T., Domitrovich, C., \& Bumbarger, B. (2001). The prevention of mental disorders in school-aged children: Current state of the field. Prevention and Treatment, 4(1) $1-62$.

Gunz, J. (1996). Jacob L. Moreno and the origins of action research. Educational Action Research, 4(1), 145-148.

Henin, A., Warman, M., \& Kendall, P.C. (2002). Cognitive behavioural therapy with children and adolescents. In G. Simos (Ed.), Cognitive behaviour therapy: A guide for the practising clinician (pp. 275-313). New York: Taylor \& Francis.

Hoag, M.J., \& Burlingame, G.M. (1997). Child and adolescent group psychotherapy: A narrative review of effectiveness and the case for meta-analysis. Journal of Child and Adolescent Group Therapy, 7(2), 51-68.

Hoff, K.E., \& Du Paul, G.J. (1998). Reducing disruptive behavior in general education classrooms: The use of self-management strategies. School Psychology Review, 27(2), 290-303.

Johnson, C.V., Riester, A.E., Corbett, C., Buehler, A., Huffaker, L., Levich, K., et al. (1998). Group activities for children and adolescents: An activity group therapy approach. Journal of Child and Adolescent Group Therapy, 8(2), 71-88.

Kaplan, J.S., \& Carter, J. (1995). Cognitive strategies: Changing beliefs, beyond behaviour modification. A cognitive-behavioural approach to behaviour management in schools (3rd ed.). Austin, TX: Pro-ed.

Kastner, J.W. (1998). Clinical change in adolescent aggressive behavior: A group therapy approach. Journal of Child and Adolescent Group Therapy, 8(1), 23-33.

Kendall, P.C., \& Panichelli-Mindel, S.M. (1995). Cognitive-behavioral treatments. Journal of Abnormal Child Psychology, 23(1), 107-124.

Little, E., \& Hudson, A. (1998). Conduct problems and treatment across home and school; A review of the literature. Behaviour Change, 15(4), 213-227.

Mishna, F., \& Muskat, B. (1998). Group therapy for boys with features of Asperger's syndrome and concurrent learning disabilities: Finding a peer group. Journal of Child \& Adolescent Group Therapy, 8(3), 97-113. 
Posthuma, B.W. (1996). Small groups in counselling and therapy: Process and leadership. Boston: Allyn \& Bacon.

Renfrew, J.W. (1997). Aggression and its causes. New York: Oxford University Press.

Schuyler, D. (2003). Cognitive therapy: A practical guide. London: W.W. Norton.

Stoiber, K.C., \& Kratochwill, T.R. (1998). Handbook of group intervention for children and families. Boston: Allyn and Bacon.

Stringer, E., \& Dwyer, R. (2005). Action research in human services. Columbus, OH: Pearson Education.

Taylor, J.L., \& Novaco, R.W. (2005). Anger treatment for people with developmental disabilities: A theory, evidence, and manual based approach. Chichester, United Kingdom: Wiley.

Walker, H.M., Severson, H.H., Feil, E.G., Stiller, B., \& Golly, A. (1998). First step to success: Intervening at the point of school entry to prevent antisocial behavior patterns. Psychology in the Schools, 35(3), 259-269.

White, J.R. (2000) Cognitive-behavioral group therapy for specific problems. In J.R. White $\&$ A.S. Freeman (Eds.), Cognitive-behavioral group therapy for specific problems and populations (pp. 3-25). Washington, DC: American Psychological Association.

Zeni, J. (1998). A guide to ethical issues and action research. Educational Action Research, 6(1), 9-19. 
Copyright of Australian Journal of Guidance \& Counselling is the property of Australian Academic Press and its content may not be copied or emailed to multiple sites or posted to a listserv without the copyright holder's express written permission. However, users may print, download, or email articles for individual use. 